Research Article

\title{
Application Study on the Dynamic Prediction Model for Determining the Mining Subsidence
}

\author{
H.F. HU ${ }^{1}$, X.G. LIAN ${ }^{1}$ and Y. $\mathrm{LI}^{2}$ \\ ${ }^{1}$ College of Mining Engineering, Taiyuan University of Technology, Taiyuan, Shanxi, 030024, China \\ ${ }^{2}$ Western Australian School of Mines, Curtin University, Kalgoorlie 6430, Australia
}

Received 6 January 2015; Accepted 24 April 2016

\begin{abstract}
The movement and deformation of the overlying strata and surface induced by underground mining are processes involving temporal and spatial changes, which, in turn, cause damage to buildings. Estimating the dynamic ground movement and deformation is important to ascertain the damage degree of buildings. To predict the dynamic ground subsidence caused by underground coal mining and protect ground buildings from dynamic damage, a dynamic prediction model was established to calculate ground deformation. A derivation of the dynamic subsidence prediction model based on Knothe's dynamic subsidence hypothesis and Litwiniszyn's stochastic medium theory was proposed under the conditions of constant advancing speed mining and post-mining. The composition and significance of the dynamic model were analyzed and provided a basis for calculating the dynamics and programming implementation. Through case studies, the dynamic prediction model was verified and the expected results were obtained during mining and post-mining. Results showed the dynamic prediction model is fit for the normal geological condition of mining but is limited for the special geological condition, that is, thick loose layer and thick bedrock. The study can meet the demand of protecting ground buildings from deformation caused by underground mining. The proposed model solves the time issue in mining subsidence prediction.
\end{abstract}

Keywords: Mining subsidence, Dynamic prediction model, Delay influence, Advancing position, Temporal spatial change.

\section{Introduction}

The movement and deformation of strata and ground caused by underground coal mining are highly complex processes affected by numerous factors. From a temporal perspective, deformation caused by mining subsidence can be divided into two types: dynamic and static surface movement. Therefore, the surface-protected object is destroyed from dynamic to static. For most buildings during the mining process, once the structure is damaged, even if the failure eventually disappears, the building will no longer be safe for use. Thus, studying the dynamic prediction model and obtaining the dynamic surface movement and deformation are critical to the goal of protecting buildings from dynamic deformation.

\section{State of the art}

Research on dynamic ground movement and deformation induced by underground coal mining around the world has been conducted for approximately 50 years. Four aspects characterize the dynamic prediction method and theory in the field of mining subsidence.

The time function of the dynamic ground movement is derived from Knothe's dynamic subsidence hypothesis. Many scholars have studied this model, including its limitations and ground subsidence prediction [1-10]. Lian

\footnotetext{
*E-mail address: tyhhf65@163.com

ISSN: 1791-2377 (C) 2016 Eastern Macedonia and Thrace Institute of Technology. All rights reserved.
}

reviewed and extended an existing classical prediction model of dynamic subsidence and proposed potential new research avenues offered by cellular automata (CA) models. The Knothe influence function model and the significance of subcritical mining geometry were analyzed. The prediction results were verified against the subsidence field survey data to assess their quality and acceptability [1]. On the basis of the Knothe time function, the computational method of timedependent surface movement for underground mining was established and the determination of time factor was discussed. The limitation of this time function was analyzed, and the reasonable distributions in accordance with practice were achieved [2]. Through theory proof and comparison with actual measurement data, the fault of the Knothe differential equation was revealed through a description of the process of surface subsidence. A more objective and reasonable expression for the time function of surface subsidence was derived and presented through an analysis of the whole process of surface subsidence; the expression was verified with actual measurement data from Dongpang mine [3]. Wang Xunchun proposed a dynamic prediction model by combining the probability integral method with the DInSAR measure technique based on the conclusion of mining subsidence prediction and the extant problems of monitoring technology. The subsidence rate and time threshold calculated by the mining dynamic prediction method could more accurately predict the level of deep mining subsidence and quantitatively estimate the regularity of damage evolution of environmental resources [4]. Xinrong Liu conducted a careful analysis on the regularity of surface subsidence in mined-out areas and proposed a new time function based on the Harris curve model by 
considering the shortage of current surface subsidence time functions [5]. The CA, a new method applied to the dynamic evolution model of coal mining subsidence, provides a new idea for the prediction research of mining subsidence [6]. $\mathrm{Wu}$ Xiao considered a long wall panel in Shandong province as a case study. Dynamic mining subsidence was simulated using mining subsidence prediction software. The topsoil removal time, scope, and topsoil depth were determined based on the simulations [7]. The time functions of surface subsidence dynamic coordinates were proposed based on the probability integration principle, the calculation methods of surface dynamic process were established, the applicability and limitations of the dynamic time function were analyzed, and some examples were verified [8]. A method to calculate the parameter of the Knothe time function was presented. The principle of predicting dynamic mining subsidence was discussed on the basis of the relationship between the periodic fractures of the main roof and surface subsidence [9]. A new time function based on Knothe's time function was proposed by adding a parameter that proved more accurate in predicting surface subsidence than the Knothe function [10].

Rheological mechanics was used to study the relationships between ground subsidence and time during underground coal mining [11-12]. On the basis of rheological theory, which discussed the surface deformation mechanism of dynamic subsidence on the assumption that both roof and coal seam are viscoelastic media, Yu Yang proposed the idea that the principle of surface deformation is similar to that of roofs, except for their parameters [11]. Yu Xueyi, based on Knothe's theory, completed the theological model on overlying strata displacement and deformation through the zero model, and analyzed the surface dynamic movement and deformation characteristics given the prediction theory and the parameters of dynamic displacement [12].

Field data were used to establish the dynamic prediction experiential function [13-15]. A new time function was proposed to cover the disadvantage of Knothe's time function in predicting surface subsidence by adding parameter $k$. The velocity and acceleration of the progressive surface subsidence obtained from this new time function were compared with actual subsidence data from an iron mine in China. This new time function was combined with the section function of a surface subsidence basin, a dynamic mode was proposed to predict surface subsidence induced by underground mining, and then a case study based on this model was conducted [13]. Several methods of mine stabilization have been used from dynamic compaction to concrete dip pillars to provide rigid in-stope support, to plugging stopes and grouting above the plug. Some case histories are provided to illustrate the subsidence problem and how it has been dealt with [14]. Published data show that the dynamic subsidence profile at low face advance rates is independent of the advance rate itself. This finding is incompatible with the common practice of modeling subsidence as a simple viscoelastic process [15].

Numerical simulation software has been used to predict the dynamic movement and deformation of the ground [1617]. A new computational method was proposed to calculate $3 \mathrm{D}$ dynamic subsidence during the process of undermining by combining stochastic theory, the Knothe model, and the geographic information system [16]. A dynamic analysis model of mining-induced subsidence was established based on the analysis on the laws of movement and deformation inside an overburdened rock mass. The presented model was applicable to predict mining-induced subsidence, analyze the movement state, and especially control the mining-induced subsidence [17].

An analysis of the above references revealed that the Knothe model is a popular method in dynamic subsidence prediction, but the solution for this model always considered the extracting space as a constant. The extracting space changes with the advance of the working face; hence, the ground dynamic subsidence increases with the elapsing time. During post-mining, the ground will reach its final subsidence after a certain time, at which point, the subsidence ratio will not increase and remain constant. As a result, this study established an entire process dynamic prediction model that includes during mining and postmining.

The remainder of this paper is organized as follows. Section 3 establishes the dynamic prediction model of mining subsidence. Section 4 analyzes the geometrical significance of the model and discusses the applicability of the model through case studies. Section 5 summarizes the conclusions.

\section{Methodology}

To establish the dynamic prediction model of mining subsidence, the quantitative relationship of subsidence with some related factors (including time-extracted size of the mining and the geology conditions) must be illustrated. This study used two views to establish the quantitative relation. One views the entire set of features of the subsidence basin. Based on the hypothesis of Knothe dynamic subsidence, the subsidence of the ground prediction points was macroscopically analyzed and a mathematic model during mining was established. The time factor based on the model was introduced to the dynamic subsidence model during post-mining. The other view is based on Litwiniszyn's stochastic medium theory. In view of the microscale model of subsidence at any time $t$ from micro-element mining, the dynamic subsidence predictive function was deduced. The different approaches of A) and B) reached the same mathematic model, which verified the correctness of the deduced model.

\subsection{Hypothesis of the model}

In 1953, the Polish scholar Knothe, on the basis of soil mechanics, proposed that the ground subsidence velocity $d W(t) / d t$ is the ratio of the difference between the final subsidence $W^{f}(t)$ and the dynamic subsidence $W(t)$ at time $t$, that is,

$$
\frac{d W(t)}{d t}=c\left[W^{f}(t)-W(t)\right]
$$

where

$d W(t) / d t=$ ground subsidence velocity,

$W^{f}(t)=$ final subsidence at time $t$,

$W(t)=$ dynamic subsidence at time $t$, and

$c=$ time influence factor.

3.2 Modeling based on the Knothe hypothesis (1) Ground subsidence dynamic prediction model during mining 
Regarding the solutions of Function (1), when $W^{f}(t)$ is a variable, the function is a linear inhomogeneous first-order differential equation and the working face is advancing. When $W^{f}(t)$ is a constant, the function is a linear homogeneous first-order differential equation and the mining stops; this condition was normally used in [2,3]. The solution in this paper is the basic function for the dynamic prediction model during mining and post-mining.

During mining, the solution of Function (1) is $W\left(x_{0}, x_{t}\right)=W^{f}\left(x_{0}, x_{t}\right)$

$-\exp \left(\frac{u^{2}}{4 \pi}+u \frac{x_{t}}{r}\right) \frac{W_{\max }}{r} \int_{x_{0}}^{x_{t}} \exp \left[-\frac{\pi}{r^{2}}\left(x+\frac{u r}{2 \pi}\right)^{2}\right] d x$

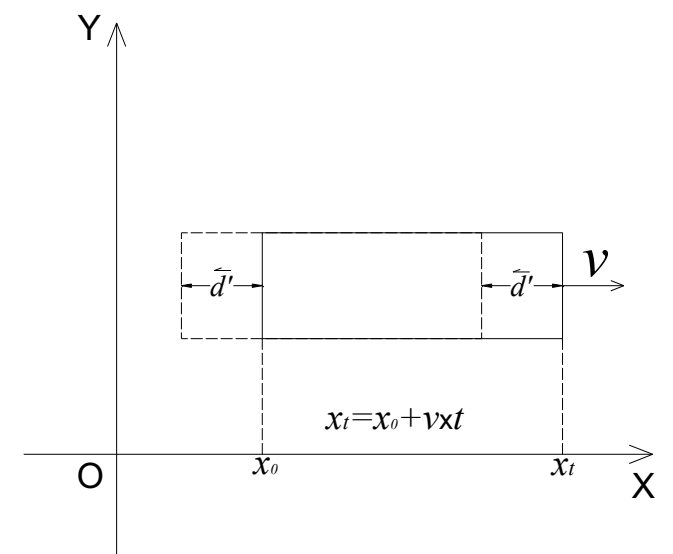

(a)the same direction of $x$-axis and $v$

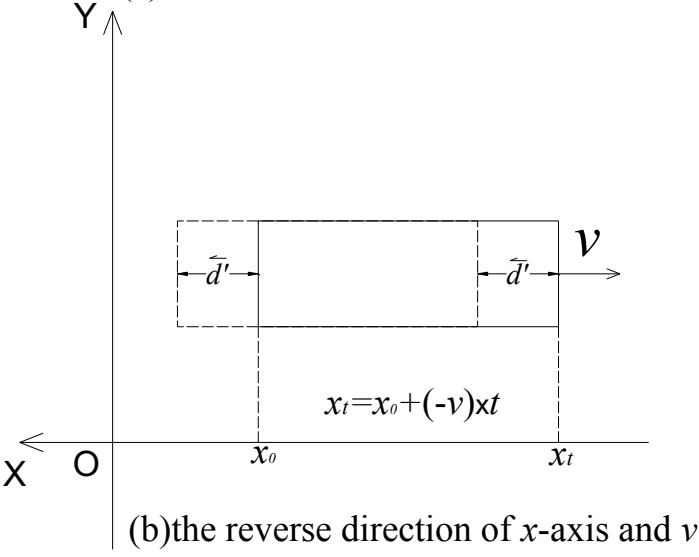

Fig. 1 Coordinate system of the dynamic ground subsidence prediction model
Two options exist for establishing the coordinate system of the dynamic subsidence prediction model, as shown in Fig. 1. Option (1): the $\mathrm{X}$-axis is in the same direction as the advancement. Option (2): the $\mathrm{X}$-axis is in the opposite direction as the advancement. The above model was established based on Figs. 1(a), and Figs. 1(b) and (c) can be utilized to establish the model for the same working face. Fig. 1(c) is shifted from Fig. 1(b).

Table 1 Variable sign of the different prediction model coordinate systems

\begin{tabular}{lccccc}
\hline Coordinate system & $v$ & $u=-\frac{c r}{v}$ & $d^{\prime}=\frac{u r}{2 \pi}$ & $x_{t}$ & $u \frac{x_{t}}{r}$ \\
\hline$x$ and $v$ same (a) & + & - & - & $+(-)$ & $-(+)$ \\
$x$ and $v$ reverse (b \& c) & - & + & + & $-(+)$ & $-(+)$ \\
\hline
\end{tabular}

Table 1 presents the variable signs of the two options to establish the prediction model. The prediction model is not changed for the two model coordinate systems. Function (1) is based on Fig. 1(a). When the x-axis reverses with the advancing direction, in Formula (2), only the upper and lower limits of the integral must be swapped, that is,

$$
\begin{aligned}
& W\left(x_{t}, x_{0}\right)=W^{f}\left(x_{t}, x_{0}\right) \\
& -\exp \left(\frac{u^{2}}{4 \pi}+u \frac{x_{t}}{r}\right) \frac{W_{\max }}{r} \int_{x_{t}}^{x_{0}} \exp \left[-\frac{\pi}{r^{2}}\left(x+\frac{u r}{2 \pi}\right)^{2}\right] d x
\end{aligned}
$$

(2) Ground subsidence dynamic prediction model during post-mining

When extraction at the working face stops, the size of the extracted material is a constant; thus, the solution of Function (1) is

$$
W(t)=W^{f} \cdot[1-\exp (-c t)]
$$

Formula (4) is the subsidence prediction model for any point at any time $t$. 
The model is improved by combining the dynamic prediction models for the cases of during mining and postmining and expanding with the model from $2 \mathrm{D}$ to $3 \mathrm{D}$; an example of the results of this model is shown in Fig. 2. Finally, the comprehensive expression of the dynamic ground prediction model for the entire mining process can be obtained as

$$
\begin{aligned}
& W\left(x_{0}, x_{t}, S_{t}, \Delta t\right)=W^{f}\left(x_{0}, x_{t}, S_{t}\right) \\
& -\exp \left(\frac{u^{2}}{4 \pi}+u \frac{x_{t}}{r}-c \Delta t\right) W^{f}\left(x_{0}+\frac{u r}{2 \pi}, x_{t}+\frac{u r}{2 \pi}, S_{t}\right)
\end{aligned}
$$

where

$$
W^{f}\left(x_{0}, x_{t}, S_{t}\right)=\text { final subsidence at time } \mathrm{t},
$$

$r=$ main radius of influence,

$v=$ advancing velocity,

$c=$ time influence factor,

$\Delta t=$ elapsed time during post-mining, and

$d^{\prime}=\frac{u r}{2 \pi}=$ delay influence distance of the working face, $u=-\frac{c r}{v}$. Let $f\left(u, \frac{x_{t}}{r}, \Delta t\right)=\exp \left(\frac{u^{2}}{4 \pi}+u \frac{x_{t}}{r}-c \Delta t\right)$, and define it as the delay factor during mining.

Formula (5) is the dynamic prediction subsidence of ground point $\mathrm{O}(0,0)$ for the extracted area $S_{t}$ at time $t$ during mining or post-mining for the time elapsed $\Delta t$, which can be simplified as

$$
\begin{aligned}
& W\left(x_{0}, x_{t}, S_{t}, \Delta t\right)=W^{f}\left(x_{0}, x_{t}, S_{t}\right) \\
& -f\left(u, \frac{x_{t}}{r}, \Delta t\right) W^{f}\left(x_{0}+d^{\prime}, x_{t}+d^{\prime}, S_{t}\right)
\end{aligned}
$$

From the mining time schedule, the meanings of Formula (6) can be illustrated as follows. During the advance of the working face, the ground prediction subsidence is expressed by two parts and $\Delta t=0$; after mining stops and time $\Delta t$ elapses, the ground subsidence is expressed by two parts; the final subsidence of the working face is expressed by the first part.

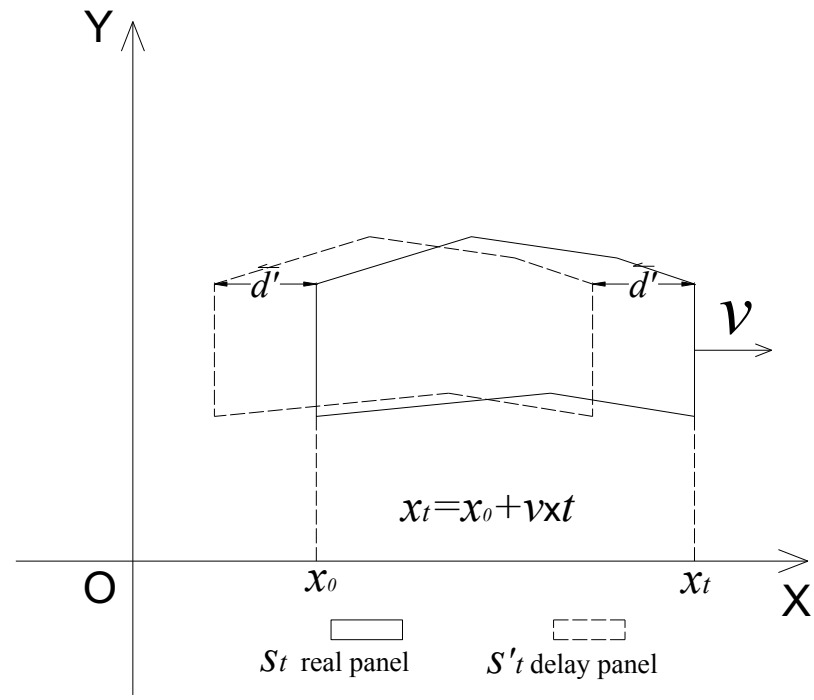

Fig. 2 Delay influence of the extracted area

Strata propagation induced by underground mining is a dynamic changing process during the advance of the working face. The delay influence distance $d$ ' can be obtained from the model and from the delay influence factor regarding the advancing distance, advancing velocity, and $\Delta t$. The final subsidence of the extracted area of delay influence is multiplied by the delay influence factor, which is the second part of Formula (6), namely, the potential subsidence.

\subsection{Modeling based on stochastic medium theory}

According to the dynamic subsidence influence function of micro-element mining based on stochastic medium theory shown in Fig. 3, the ground subsidence influence expression is

$$
W_{e}(t)=\left(1-e^{-c t}\right) \frac{1}{r} e^{-\pi \frac{x^{2}}{r^{2}}}
$$

At time $t$ during the advance and after mining stopped at time $\Delta t$, the micro-element mining subsidence expression is

$$
\begin{aligned}
& W_{e}\left(x_{t}\right)=\frac{1}{r} \exp \left(-\pi \frac{x^{2}}{r^{2}}\right) \\
& -\exp \left(\frac{u^{2}}{4 \pi}+u \frac{x_{t}}{r}-c \Delta t\right) \frac{1}{r} \exp \left(-\pi \frac{\left(x+\frac{u r}{2 \pi}\right)^{2}}{r^{2}}\right)
\end{aligned}
$$

Formula (8) is expanded to three dimensions, integrated over the extracted area $S t$, and multiplied by the maximum subsidence $W_{\max }$, which provides the same result as Formula (6).

\section{Result Analysis and Discussion}

\subsection{Geometrical significance of the model}

The corresponding curves of every part of Formula (6), shown in Fig. 4, are as follows:

$$
\begin{aligned}
& W^{f}\left(x_{0}, x_{t}, S_{t}\right)=\text { curve } 1 \text { is the final subsidence, } \\
& W^{f}\left(x_{0}+d^{\prime}, x_{t}+d^{\prime}, S_{t}\right)=\text { curve } 2 \text { is the delay influence of }
\end{aligned}
$$
the final subsidence,

$$
f\left(u, \frac{x_{t}}{r}, \Delta t\right)=\exp \left(\frac{u^{2}}{4 \pi}+u \frac{x_{t}}{r}-c \Delta t\right)=\text { curve } 5 \text { is the delay }
$$

influence factor,

$$
f\left(u, \frac{x_{t}}{r}, \Delta t\right) W^{f}\left(x_{0}+d^{\prime}, x_{t}+d^{\prime}, S_{t}\right)=\text { curve } 3 \text { is the }
$$

potential subsidence, and

$$
W\left(x_{0}, x_{t}, S_{t}, \Delta t\right)=\text { curve } 4 \text { is the dynamic subsidence. }
$$

Note that the above dynamic prediction model is developed based on constant velocity mining and for the inconstant velocity of mining, which can be segmented into different constant velocity mining segments.

\subsection{Case study A}

A coal mine panel in Anhui Province, China, with a strike length of $620 \mathrm{~m}$, dip width of $162 \mathrm{~m}$, and average mining depth of $500 \mathrm{~m}$, including a loose layer of $400 \mathrm{~m}$ and a bedrock layer of $100 \mathrm{~m}$, was considered in this study. The extraction method was backing a long wall with full mechanization, and a caving method for the roof. The mining coal height was $3 \mathrm{~m}$, and the advancing velocity was $56 \mathrm{~m} /$ month. According to the comparison of prediction results and the field data shown in Fig. 5, the accuracy of this dynamic model is good during the working face advance and after mining stopped. 


\subsection{Case study B}

The application coal mine panel lies in Anhui Province, China, similar to the case above. The mining depth was 780 $\mathrm{m}$, which consisted of $437 \mathrm{~m}$ of loose layer and $343 \mathrm{~m}$ of bedrock. Mining height was $3.3 \mathrm{~m}$, angle of inclined coal seam was $5^{\circ}$, longitudinal length was $2613 \mathrm{~m}$, and transverse length was $240 \mathrm{~m}$. Fully mechanized long wall retreating mining was used, and the entire roof collapsed. Fig.6 shows the dynamic surface subsidence troughs of the panel in the Huainan mining area. Results have sufficient accuracy, with the maximum error at $10 \%$ of the maximum subsidence.

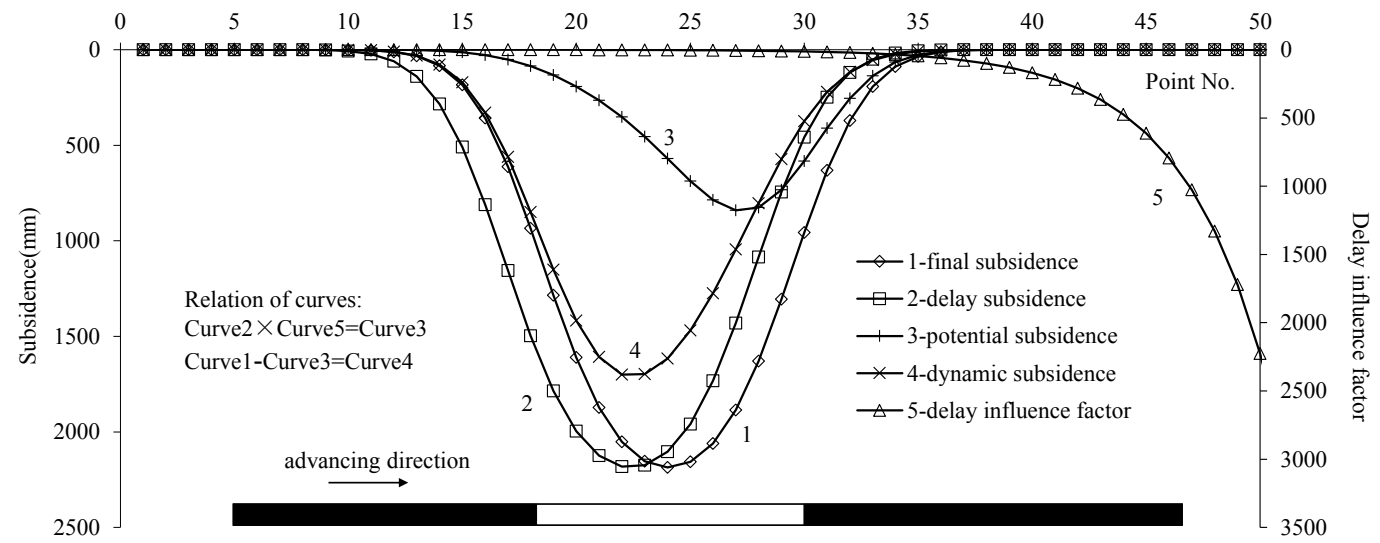

Fig. 4 Geometrical significance of dynamic subsidence
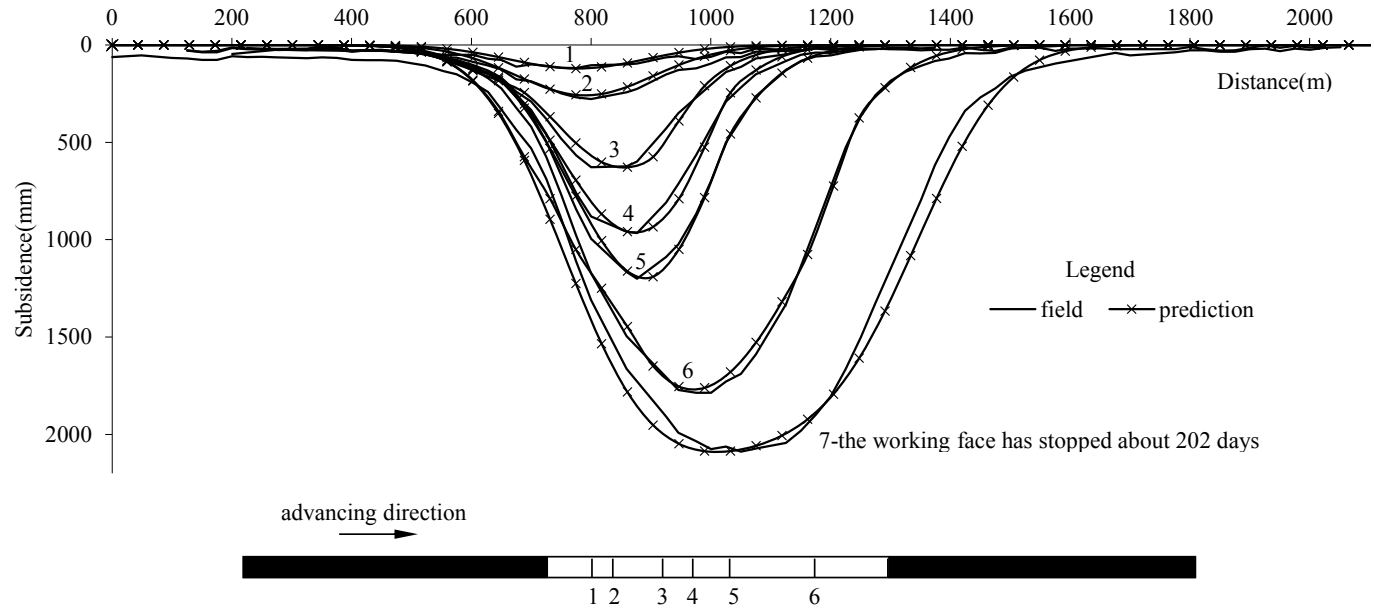

Fig. 5 Results comparing the prediction and the experimental subsidence curves

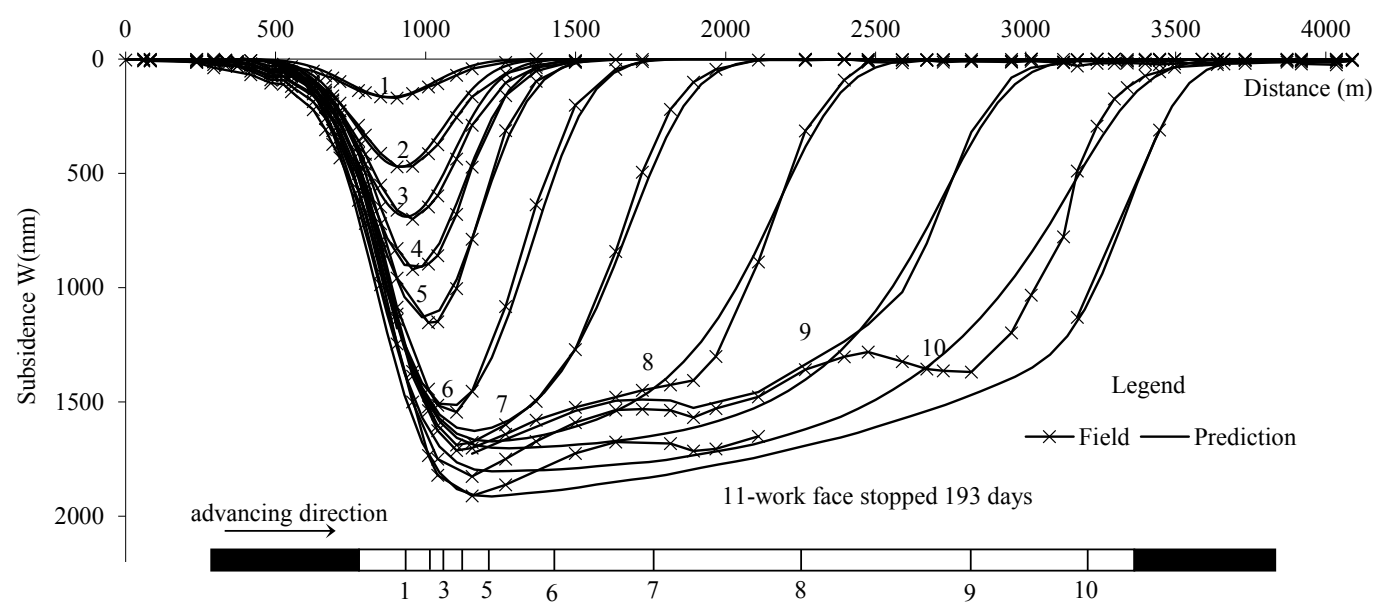

Fig. 6 Surface subsidence troughs of a panel in advancing

\subsection{Limitation of the model}

As per the hypothesis, the model is established based on stochastic medium theory, which requires all rocks to be isotropic and uniformly medium. However, this condition is impossible for the strata of different mining areas, which are under different geological conditions, especially when the overlying strata consist of thick loose layer and bedrock. The main predicting problem for this special geological condition is not consistent at the boundary of the subsidence curves. 


\section{Conclusions}

To predict the dynamic deformation and movement of ground caused by underground coal mining, and protect surface buildings from dynamic deformation, the Knothe model and stochastic medium theory were respectively used as basis to establish the dynamic subsidence prediction model. The conclusions were obtained as follows.

(1) Based on the hypothesis of Knothe dynamic subsidence and the stochastic medium theory micro-model, the dynamic prediction model for the entire mining process was deduced, and the correctness of the model from the macroscopic and micro-scale views was verified.

(2) The delayed influence of mining subsidence was considered through the time-space transfer between the advancing position and the delay time to obtain the dynamic prediction model in the advance of the working face. The subsidence prediction was introduced in instantaneous mining for a working face, and the obtained subsidence dynamic prediction model during post-mining was used to establish the dynamic prediction model for during mining and post-mining.

(3) The case studies considered in this study verified that the dynamic prediction model and method can reasonably describe ground dynamic subsidence. For the case of inconstant velocity mining, this method can be segmented into smaller constant mining segments for modeling.

This study can meet the demands for ground protection plans in the coal mine industry and protect buildings from dynamic damages. However, the model is limited by special geological conditions, such as strata consisting of thick loose soil and thin bedrock. Further study is needed to solve the prediction model in special geological conditions.

\section{Acknowledgements}

The study was supported by the National Natural Science Foundation of China (51574132 and 51504159) and the Natural Science Foundation of Shanxi Province, China (2014011001-3).

\section{References}

1. Xu-gang LIAN, Andrew JAROSZ, Jose SAAVEDRA-ROSAS et al, "Extending dynamic models of mining subsidence". Transactions of Nonferrous Metals Society of China, 21(S3), 2011, pp.s536-s542.

2. Cui Ximin, Miao Xiexing, Zhao Yingli et al. "Discussion on the time function of time dependent surface movement". Journal of China Coal Society, 36(05), 1999, pp.453-456.

3. Chang Zhanqiang, Wang Jinzhuang, "Study on time function of surface subsidence the improved Knothe time function". Chinese Journal of Rock Mechanics and Engineering, 22(09), 2003, pp.14961499.

4. Wang XunChun, Zhang Yue, Jiang XingGe, et al, "A Dynamic Prediction Method of Deep Mining Subsidence Combines D-InSAR Technique". Procedia Environmental Sciences, 2011, pp.2533-2539.

5. Xinrong Liu, Junbao Wang, Jianqiang Guo et al, "Time function of surface subsidence based on Harris model in mined-out area". International Journal of Mining Science and Technology, 23(2), 2013, pp.245-248.

6. Xue Yang Sun, Yu Cheng Xia, "Dynamic Prediction Model of Mining Subsidence Based on Cellular Automata". Advanced Materials Research, 962-965, 2014, pp.1056-1061.

7. Wu Xiao, Zhenqi Hu, Yoginder P. Chugh et al, "Dynamic subsidence simulation and topsoil removal strategy in high groundwater table and underground coal mining area: a case study in Shandong Province". International Journal of Mining, Reclamation and Environment, 28(4), 2014, pp.250-263.

8. Ying Guo, Guang Yi Zhu, Xin Liang Jiang et al, "Study of Dynamic Coordinates Time Function in Mine Subsidence". Advanced Materials Research, 594-597, 2012, pp.56-60.
9. Yin-Xiao Ji, Meng-Ru Wang, Guo Wang et al, "Key Technology of Predicting Dynamic Surface Subsidence Based on Knothe Time Function”. Journal of Software, 6(7), 2011, pp.1273-1280.

10. Hao Liang Han, Bo Cui, "Modeling of Surface Subsidence Based on Time Function". Advanced Materials Research, 422, 2011, pp.318321.

11. Yu Yang, Zhiqiang Gong, Bing Liang, "Dynamic subsidence basins in coal mines based on rock mass rheological theory". Mining Science and Technology (China), 21(3), 2011, pp.323-327.

12. Yu Xueyi, Dang Tianhu, Pan Hongyu et al, "Theological characteristics of surface dynamic subsidence by mining". Journal of Xi'An University of Science and Technology, 23(02), 2003, pp.131-134. 13. Hao Liang Han, Nai Lian Hu, Bo Cui, "Dynamic Modeling of Surface Subsidence Induced by Underground Mining". Advanced Materials Research, 382, 2011, pp.323-327.

14. T. R. Stacey and F. G. Bell. "The influence of subsidence on planning and development in Johannesburg, South Africa". Environmental and Engineering Geoscience, 5(04), 1999, pp.373-388.

15. Goulty, NR, AlRawahy, SYS, "Reappraisal of time-dependent subsidence due to longwall coal mining". Quarterly Journal of Engineering Geology, 29(1), 1996, pp.83-91.

16. Ibrahim Djamaluddin, Yasuhiro Mitani, Hiro Ikemi, "GIS-Based Computational Method for Simulating the Components of 3D Dynamic Ground Subsidence during the Process of Undermining”. International Journal of Geomechanics, 12(01), 2012, pp.43.

17. Li Xinqiang, Gao Yanfa, Zhang Qingsong, "Study on dynamic numerical simulation of mining-induced subsidence". Chinese Journal of Rock Mechanics and Engineering, 23(01), 2004, pp.86-90. 